\section{MICROGLIA}

\section{Heterogeneity in the retina \\ Immunity 50, 723-737 (2019)}

The density, morphology and marker expression of microglia vary by brain region. In Immunity, Saban and colleagues characterize distinct classes of microglia in the retina at steady state and during degenerative diseases. The authors show that $85 \%$ of retinal microglia are yolk-sack derived and long-lived. Microglia in the inner plexiform layer of the retina, but not those in the outer plexiform layer, are dependent on the microglia maintenance factor IL-34. In models of acute or chronic photoreceptor degeneration, microglia from the inner and outer plexiform layers, but not monocyte-derived macrophages, migrate to the subretinal space, where they downregulate genes encoding homeostatic molecules (e.g., Tmem119, Selplg and CX3cr1) and upregulate genes encoding molecules in the cell-adhesion, lipidmetabolism and antioxidant pathways. IL-34-deficient mice have defects in cone photoreceptor responses at steady state and have increased damage to the retinal pigmented epithelium during photoreceptor degeneration, possibly owing to loss of deadphotoreceptor clearance.

IV

https://doi.org/10.1038/s41590-019-0390-5

\section{SIGNAL TRANSDUCTION}

\section{Signaling platforms}

Cell https://doi.org/10.1016/j.cell.2019.01.039 (2019)

The detection of microbes by Toll-like receptors (TLRs) engages either a large oligomeric protein complex dependent on the adaptor MyD88 (myddosome) that activates the inflammatory transcription factors NF- $\kappa$ B and AP- 1 or a complex dependent on the TLR adaptor TRIF that activates the kinase TBK1 to induce type I interferons. In Cell, Tan and Kagan show that TBK1 is a component of the myddosome and is required for the induction of aerobic glycolysis. TLR ligands induce glycolysis in macrophages independently of transcription and with dependence on MyD88 and TBK1. TBK1 is recruited to the myddosome by the adaptor TRAF6 independently of TRIF. Proving the modular nature of the myddosome, MyD88-RIP3 chimeric molecules can induce necroptosis, and MyD88-NpLxIS, which contains an IRF3-activation motif, can activate type I interferons, while a synthetic construct that can oligomerize and contains the NpLxIS motif also activates type I interferons. This indicates that supramolecular organizing centers, such as the myddosome and the inflammasome, are modular machines made of two functional units: an oligomerization platform and an effector domain.

https://doi.org/10.1038/s41590-019-0391-4

\section{NLRP1 INFLAMMASOMES}

\section{Functional degradation}

Science https://doi.org/10.1126/science.aau1330 \& https://doi.org/10.1126/science.aau1208 (2019)

Pathogens such as Bacillus anthracis and Shigella flexneri encode factors that trigger activation of NLRP1 inflammasomes. In Science, Sandstrom et al. and Chui et al. reveal that NLRP1 is an intracellular decoy

\title{
REPORTER SYSTEMS
}

Macrophages light the way

Biomarkers have great potential for the early detection of disease but can suffer drawbacks in their sensitivity and specificity. In Nature Biotechnology, Gambhir and colleagues exploit the functional plasticity and tumor-homing ability of macrophages to engineer a cellular reporter that can diagnose the presence of cancer in a highly sensitive way. Within the tumor microenvironment, macrophages reliably switch to an 'M2-like' phenotype characterized most clearly by their expression of arginase-1. Macrophages engineered to express and release Gaussia luciferase after upregulation of arginase-1 can report the presence of even very small tumors $\left(<50 \mathrm{~mm}^{3}\right)$, including those that are below the detection limits of positron-emission tomography scanning. Reporter activity occurs only in an anti-inflammatory tumor microenvironment. This cellular reporter approach might be applicable for the diagnosis of variety of disease states.

protein whose $\mathrm{N}$-terminal domain requires proteasomal degradation for inflammasome activation. Mouse NLRP1 and human CARD8 have a C-terminal caspaserecruitment domain (CARD) preceded by a 'function-to-find' domain that undergoes constitutive autoprocessing to yield noncovalently linked NLRP1 fragments. B. anthracis lethal toxin directly cleaves the $\mathrm{N}$-terminal portion of NLRP1, whereas the $S$. flexneri-encoded E3 ubiquitin ligase IpaH7.8 modifies NLRP1. Both processes lead to proteasome-mediated degradation of the N-terminal NLRP1 domain, which releases the $\mathrm{C}$-terminal CARD to activate caspase-1 and trigger cellular pyroptosis. Chui et al. provide genetic evidence of host-encoded E3 ligases of the $\mathrm{N}$-end rule proteasomal degradation pathway in lethal toxin-initiated activation of NLRP1. Thus, proteasomal targeting of NLRP1 leads to its functional activation.

https://doi.org/10.1038/s41590-019-0392-3

\section{CELL TRAFFICKING}

\section{Distinct S1PR roles \\ Sci. Immunol. 4, eaav1263 (2019)}

Sphingosine 1-phosphate (S1P) regulates lymphocyte trafficking. In Science Immunology, Xiong et al. show that S1P receptors (S1PRs) serve distinct roles during the migration of $\mathrm{CD} 4^{+} \mathrm{T}$ cells across lymphatic endothelial cell (LEC) barriers into the afferent lymphatics. LECs express S1PR2, which responds to S1P by upregulating expression of the adhesion molecule VCAM-1 and altering expression of cellular junction proteins, including VE-cadherin, occludin and zonulin-1. Mouse and human CD4 ${ }^{+}$ effector and memory T cells express S1PR1 and S1PR4. Inhibition or disruption of S1PR1, S1PR4 or S1PR2 diminishes the migration of $\mathrm{T}$ cells to S1P both in vitro and in vivo, but not their migration to the chemokine receptor CCR7 ligand CCL19. Inhibition of S1P signaling blunts early interactions of T cells with LECs, although S1PR1 seems to function differently from S1PR4. Interestingly, S1P-dependent signaling preferentially affects the transcellular migration of T cells through LECs rather than their paracellular trafficking. Thus, S1P governs distinct steps in the trafficking of T cells into the afferent lymphatics.

https://doi.org/10.1038/s41590-019-0393-2

Laurie A. Dempsey, Zoltan Fehervari and loana Visan 